\title{
Des lymphokines régulatrices de la production des classes d'anticorps
}

Les différents isotypes d'immunoglobuline stimulent la synthèse par les lymphocytes $T$ de récepteurs membranaires spécifiques, appelés IBF (immunoglobulin binding factors). Ces IBF qui pourraient représenter une forme soluble des récepteurs membranaires, régulent à leur tour la synthèse de l'isotype considéré par les lymphocytes B.

\section{Catherine Sautès}

Directeur de Recherche à l'Inserm.

\section{Wolf-Herman Fridman}

Directeur de Recherche à l'Inserm Directeur Unité Inserm immunologie cellulaire et clinique U255.

\section{ADRESSES}

C. Sautès, W.H. Fridman : Laboratoire d'immunologie cellulaire et clinique, Unité Inserm U.255, Institut Curie, 26, rue d'Ulm, 75005 Paris.

$m / s n^{\circ} 3$ ool. 3 , mars 87 es molécules d'anticorps peuvent être regroupées en cing classes (ou isotypes) d'immunoglobulines, codées par des gènes différents : $\operatorname{Ig} M, \operatorname{Ig} D, \operatorname{IgG}, \operatorname{IgE}$, IgA. Ces classes exercent des fonctions différentes : les $\operatorname{IgM}$ et les IgG sont impliquées dans les protections anti-bactériennes et anti-virales, les $\operatorname{IgD}$ sont des récepteurs lymphocytaires, les IgE sont les molécules responsables des allergies et jouent un rôle fondamental dans les réponses aux parasites, les $\operatorname{IgA}$ sont les anticorps des sécrétions. Toutes les immunoglobulines sont produites par une sous-population de lymphocytes, les lymphocytes B. En réponse à un antigène, le niveau de production des immunoglobulines est sous le contrôle de lymphocytes $T$, qui agissent par l'intermédiaire de lymphokines.

Les premières expériences suggérant que les anticorps circulants contrôlent leur propre synthèse ont été réalisées en 1968 par Claudia Henry et Niels Jerne [1]. Ces auteurs montrèrent que, lors d'une réponse secondaire vis-à-vis de Globules Rouges de Mouton
(GRM), l'injection de petites quantités d'anticorps de classe IgG (IgG anti-GRM) en même temps que l'antigène, réduit considérablement la production d'anticorps. Trois ans plus tard, N. Sinclair et al. reproduisirent ce phénomène et mirent en évidence l'importance de la portion Fc de l'anticorps dans cette régulation. Ils montrèrent que des fragments $\mathrm{F}(\mathrm{ab})_{2}{ }_{2}$ préparés par coupure enzymatique des IgG anti-GRM sont incapables d'exercer un effet inhibiteur, à la fois in vitro et in vivo [2].

$\mathrm{Si}$ déjà à cette époque certains auteurs soupçonnaient l'importance des lymphocytes $\mathrm{T}$ dans cette régulation, ce n'est que dix ans plus tard, en 1981, que l'on comprit réellement leur mécanisme d'action. R.D. Lynch et al. montrèrent que l'interaction de lymphocytes $\mathrm{T}$ avec des $\operatorname{Ig} \mathrm{A}$ les rend capables de supprimer la production d'IgA par des cellules de myélome [3]. L'effet est induit par l'isotype, spécifique de cet isotype et applicable à tous les isotypes. Il devint alors évident qu'une lymphokine découverte en 1974 par W.H. Fridman et al. était un élément clef de cette 
régulation [4]. Il avait été montré que les lymphocytes $\mathrm{T}$ exprimant des récepteurs pour la portion Fc des IgG sécrètent un facteur qui se lie spécifiquement au fragment Fc des IgG et supprime la production d'anticorps de classe IgG [5]. Ce facteur fut appelé IgG-Binding Factor (IgG-BF). $A$ la suite de ces travaux, des Immunoglobulin-Binding Factors (IBF) ont été décrits pour tous les isotypes d'Ig : IgG-BF, IgE-BF, $\operatorname{Ig} \mathrm{A}-\mathrm{BF}$ et récemment $\operatorname{Ig} \mathrm{D}-\mathrm{BF}$. Ils peuvent être détectés dans le sérum des souris en dehors de toute immunisation. Ainsi, chaque classe d'anticorps contrôle son propre niveau de synthèse par une boucle de régulation faisant intervenir un IBF spécifique de cette classe et dont l'activité peut être suppressive ou amplificatrice (voir la figure 1 ci-dessous).
Des perturbations des taux de lymphocytes $T$ exprimant des récepteurs $\mathrm{Fc}$ et de la sécrétion d'IBF sont associées chez l'homme à des dérèglements des taux d'Ig sériques. Le syndrome d'hyper-IgE dans lequel on trouve un taux élevé d'IgE circulante est accompagné d'un nombre élevé de cellules $\mathrm{T}$ à récepteur pour les IgE qui produisent un IgE-BF potentiateur; dans la maladie de Berger, néphropathie accompagnée d'un taux sérique élevé d'IgA, on trouve un grand nombre de cellules $\mathrm{T}$ à récepteur pour les IgA.

A côté de la régulation spécifique de l'antigène exercée par l'antigène lui-même ou par la portion idiotypique de l'anticorps, la régulation isotypique, exercée par la portion Fc de l'anticorps représente un élément majeur de réguwith components of the lymphoid system. Immunology $1971 ; 21$ : 967-71.

3. Hoover RG, Gebel HM, Dieckgraefe BK, et al. Occurrence and potential significance of increased numbers of $T$ cells with Fc receptors in myeloma. Immunol Rev 1981; 56 : 115-39.

4. Fridman WH, Golstein P. ImmunoglobulinBinding Factor present on and produced by thymus-processed lymphocytes ( $\mathrm{T}$ cells). Cell Immunol $1974 ; 11$ : 442-55.

5. Gisler RH, Fridman WH. Inhibition of the in vitro $19 \mathrm{~S}$ and $7 \mathrm{~S}$ antibody response by Immunoglobulin-Binding Factor (IBF) from alloantigen-activated $\mathrm{T}$ cells. Cell Immunol 1976 ; 23 : 99-117.

6. Néauport-Sautès $C$, Daēron $M$, Teillaud JL, Blank U, Fridman WH. The occurrence, structural and functionnal properties of Immunoglobulin Fc receptors on murine neoplastic cells. International Revizws of Immunology 1986 ; $1: 237-71$.

7. Daēron M, Yodoi J, Néauport-Sautès C, Moncuit J, Fridman WH. Receptors for immunoglobulin isotypes (FcR) on murine $T$ cells. I. Multiple FcR expression on $T$ lymphocytes and hybridoma $\mathrm{T}$ cell clones. Eur $J$ Immunol 1985 ; 15 : 662-77.

8. Anderson CL, Looney RJ. Human leukocyte IgG Fc receptors. Immunol Today 1986 ; $7: 264-6$.

9. Hibbs ML, Walker ID, Kirszbaum L, at al. The murine Fc receptor for immunoglobulin : purification, partial amino acid sequence and isolation of cDNA clones. Proc Natl Acad Sci USA 1986 ; 83 : 69804.

10. Yodoi J, Ishizaka $\mathrm{T}$, Ishizaka $\mathrm{K}$. Lymphocytes bearing Fc receptors for IgE. II. Induction of Fc receptor bearing rat lymphocy-

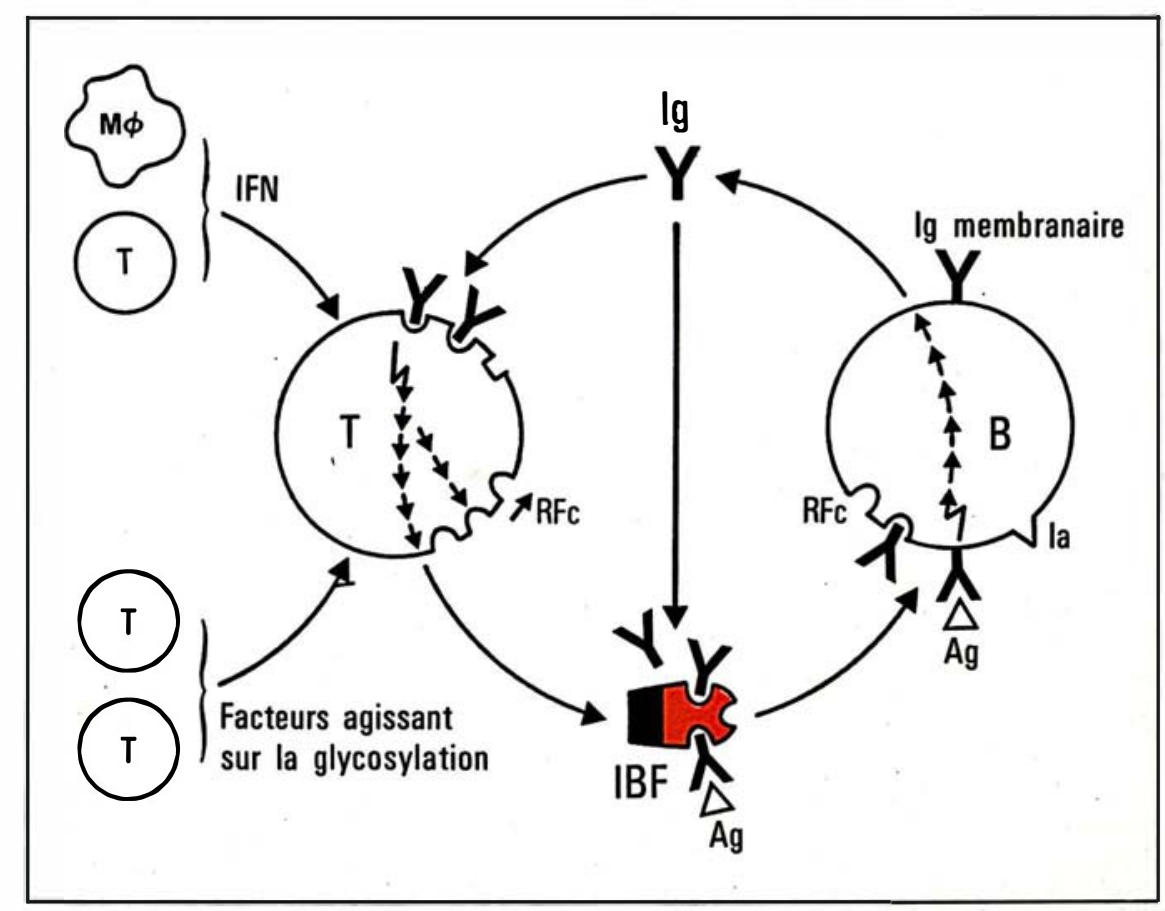

Figure 1. Boucle de régulation. Les lymphocytes $B$ sécrètent des anticorps (Ac) d'un isotype donné ( $)$. Ces Ac interagissent avec les RFc de spécificité isotypique correspondante exprimés sur les lymphocytes T. Cette interaction provoque une augmentation du taux d'expression de ces RFc et une production d'IBF spécifique de cet isotype, lequel agit au niveau des lymphocytes $B$ et régule la production de cet isotype. $\mathrm{Ig}=$ immunoglobuline $; T=$ cellule $T ; B=$ cellule $B ;$ IFN = interféron ; RFC = récepteur reconnaissant le fragment Fc des immunoglobulines $; A g=$ antigène $; \mathrm{la}=$ antigène de classe II du complexe majeur d'histocompatibilité. $M \phi=$ macrophage.

$m / s$ n 3 ool. 3, mars 87 
lation interne du système immunitaire.

Nous ferons le point des connaissances actuelles sur le rôle des lymphocytes $T$ et des IBF dans cette régulation.

\section{Les récepteurs de Fc}

Les cellules du système immunitaire expriment des récepteurs pour la portion Fc des immunoglobulines (RFc) [6]. Ces récepteurs peuvent être détectés à l'aide de différentes techniques, par exemple, par formation de rosettes entre les cellules du système immunitaire et des globules rouges recouverts d'Ig par réaction antigène-anticorps (Ig anti-GRM) (figure 2). D'autres techniques utilisent des Ig solubles, agrégées par la chaleur ou sous forme de complexes, couplées à des fluorochromes ou à des substances radioactives.

Des RFc sont exprimés sur toutes les populations lymphocytaires : lymphocytes $\mathrm{B}$, cellules NK (cellules tueuses naturelles), cellules $\mathrm{K}$ (cellules responsables de la cytotoxicité cellulaire dépendante d'anticorps) et une fraction des lymphocytes T. Sur ces derniers, l'expression des RFc est liée à l'activation et à la différenciation des cellules, les thymocytes exprimant peu ou pas de RFc alors que les cellules $\mathrm{T}$ activées expriment des taux plus importants.

Des récepteurs de $\mathrm{Fc}$ ont été décrits pour tous les isotypes d'Ig : IgG (RFc $\gamma)$, IgA (RFc $\alpha$ ), $\operatorname{IgE}(\mathrm{RFc}), \operatorname{IgM}(\mathrm{RF} c \mu)$ et $\operatorname{IgD}$ (RFc $\delta$ ) (Tableau I). A l'exception du RFc commun aux IgE et aux IgG $(\mathrm{RF} \gamma / \epsilon)$ ) présents sur certains lymphocytes B et sur les macrophages, les récepteurs de Fc sont spécifiques de la classe d'Ig. Ainsi, seules les IgG et non les $\operatorname{Ig} A, \operatorname{IgE}, \operatorname{Ig} D$ ou $\operatorname{Ig} M$ vont se fixer au RFc $\gamma$ et inhiber la formation de rosettes entre les cellules du système immunitaire et les globules rouges recouverts d'IgG (figure 2). La spécificité du RFcy pour les sous-classes d'IgG varie selon le type cellulaire. Chez la souris par exemple, il existe un récepteur commun aux IgG1, $m / s n^{\circ} 3$ vol. 3, mars 87

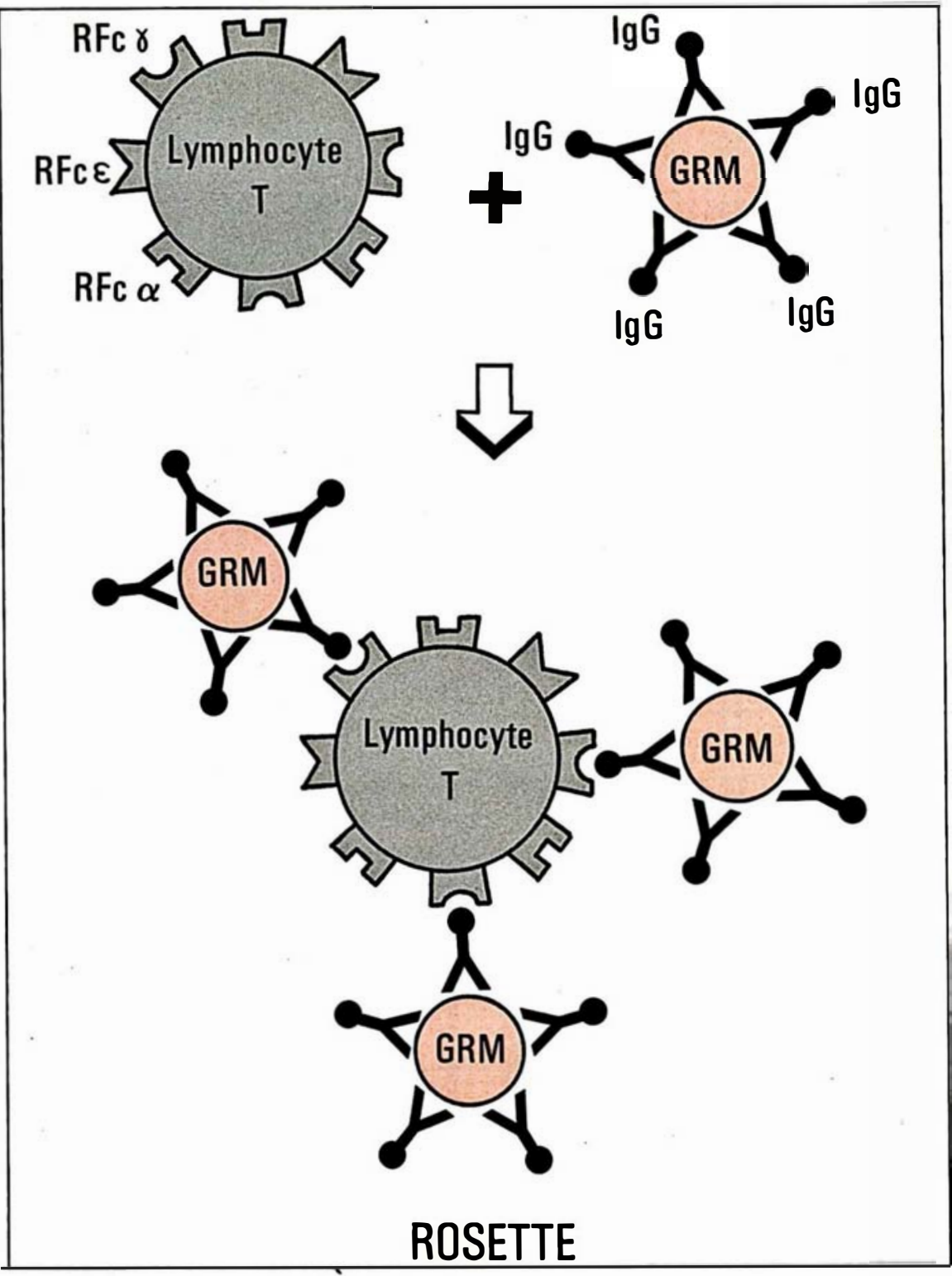

Figure 2. Détection de RFcy par formation de rosette entre un Iymphocyte $T$ et des globules rouges recouverts d'IgG. Lorsqu'un lymphocyte $T$, exprimant des RFc $\gamma$, des RFce et des $R F c \alpha$, est mis en contact avec des globules rouges de mouton (GRM) recouverts d'IgG anti-GRM, les $R F c \gamma$ se lient à la portion Fc des anticorps. Le lymphocyte $T$ se trouve entouré de plusieurs GRM, formant ainsi une rosette.

IgG2a et IgG2b sur les lymphocytes $B$, un récepteur pour les IgG2a (RFcI) distinct de celui pour les IgG1 et IgG2b (RFcII) sur les macrophages. Les lymphocytes $\mathrm{T}$ expriment à la fois un récepteur de spécificité identique à celui exprimé sur les lymphocytes $B$ et un récepteur de même spécificité que le RFcII des macrophages. La question de savoir si une population cellulaire exprime un seul ou plusieurs type(s) de RFc a été abordée sur des clones et des lignées cellulaires. Il apparaît maintenant de façon claire qu'un lymphocyte ou un macrophage peut exprimer 


\begin{tabular}{|c|c|c|}
\hline \multicolumn{3}{|c|}{$\begin{array}{c}\text { Tableau I } \\
\text { SPÉCIFICITÉ ISOTYPIQUE DES RFc DE SOURIS }\end{array}$} \\
\hline Isotype & RFc & Type cellulaire \\
\hline $\lg M$ & $\mathrm{RFc} \mu$ & $\begin{array}{l}\text { Lymphocytes T, B } \\
\text { Macrophages }\end{array}$ \\
\hline $\lg D$ & $\operatorname{RFc} \delta$ & Lymphocytes $T$ \\
\hline $\lg E$ & $\begin{array}{c}\text { RFce } \\
\begin{array}{l}\mathrm{RFc}_{\gamma} 1 / \gamma 2 \mathrm{~b} / \epsilon \\
\mathrm{RFc} \gamma 1 / \epsilon\end{array}\end{array}$ & $\begin{array}{l}\text { Lymphocytes T, B } \\
\text { Macrophages } \\
\text { Mastocytes } \\
\text { Macrophages } \\
\text { Lymphocytes } \\
\text { ganglionnaires }\end{array}$ \\
\hline $\lg A$ & RFc $\alpha$ & Lymphocytes T, B \\
\hline $\lg G$ & $\begin{array}{c}\mathrm{RFc} \gamma 1 / \gamma 2 \mathrm{a} / \gamma 2 \mathrm{~b} \\
\mathrm{RFc} \gamma 1 / \gamma 2 \mathrm{~b} \\
\mathrm{RFc} \gamma 2 \mathrm{a}\end{array}$ & $\begin{array}{l}\text { Lymphocytes T, B } \\
\text { Macrophages } \\
\text { Lymphocytes T } \\
\text { Macrophages }\end{array}$ \\
\hline
\end{tabular}

\section{RÉFÉRENCES}

11. Daēron M, NÉauport-Sautès C, Yodoi J, Moncuit J, Fridman WH. Receptors for immunoglobulin isotypes ( $F c R$ ) on murine $T$ cells. II. Multiple FCR induction on hybridoma $T$ cell clones. Eur J Immunol 1985; 15 : 668-74.

12. Fridman WH, Gresser I, Bandu MT Aguet $M$, NÉauport-Sautès $C$. Interferon enhances the expression of Fc receptors. $J$ Immunol 1980 ; 124 : 2436-41.

13. Fridman WH, Teillaud JL, Amigorena S, et al. The isotypic circuit : Immunoglobulins, Fc Receptors and Immunoglobulin-Binding Factors. Int Rev Immunol 1986; 2 : 19-38.

14. Löwy I, Brézin C, Néauport-Sautès C, Thèze J, Fridman WH. Isotype specific regulation of antibody production : $\mathrm{T}$ cell hybrids can be selectively induced to produce $\mathrm{IgGl}$ and IgG2 subclass specific suppressive Immunoglobulin-Binding Factors. Proc Natl Acad Sci USA $1983 ; 80$ : 2323-7.

15. NÉauport-Sautès C, Dupuis C, Fridman WH. Specificity of Fc Receptors of activated $T$ cells. Relation with released Immunoglobulin-Binding Factor. Eur $J$ Immunol 1975 ; 5 : 849-54.

16. Ishizaka K. Regulation of IgE synthesis. Annal Review of Immunology 1984 ; 2 : 159-82.

17. Martens CL, Huff TF, Jardieu $P$, at al. cDNA clones encoding IgE-Binding Factors from a rat-mouse T cell hybridoma. Proc Nall Acad Sci USA $1985 ; 82$ : 2460-4.

18. Lê Thi Bich-Thuy, Samarut C, Rabourdin-Combe C, Revillard JP. The suppressive activity of Fc Receptors is not related to their T cell origin. Cell Immunol 1982 ; $62: 252-62$.

19. Teillaud JL, Amigorena S, Moncuit J, et al. Regulatory effects of ImmunoglobulinBinding Factors (IBF) on hybridoma B cells. (Soumis pour publication 1986.)

20. Lê Thi Bich-Thuy, Revillard JP. Suppression of polyclonal B cell activation by IgGBinding Factors. Requirement of $\mathrm{T}$ cells. Eur $J$ Immunol 1985 ; 15 : 96-9.

21. Fridman $W H$, Daēron $M$, Amigorena $S$, Rabourdin-Combe C, NÉauport-Sautès C. Bases for an isotypic network. Mol Immunol plusieurs types de RFc (Tableau I). Par exemple chez la souris, notre laboratoire a montré que la même cellule $\mathrm{T}$ clonée exprime à la fois des RFc $\gamma$, des RFc $\alpha$, des RFc $\epsilon$ et des RFc $\mu$ [7]. L'affinité des Ig pour les RFc varie selon l'Ig. En ce qui concerne la souris, le RFc qui a la plus forte affinité est le RFcI (Kaff $=10^{8}$ litres $/ \mathrm{mole}$ ), le RFCII ayant une affinité pour les IgG cent fois plus faible [6].

Des anticorps monoclonaux (AcM) contre les RFc $\gamma$ et les RFce ont été préparés et sont à l'heure actuelle largement utilisés pour purifier les protéines et cloner les gènes des RFc. Chez l'homme, trois types de RFc différents ont été mis en évidence à l'aide d'AcM. Le RFcI est un récepteur à forte affinité $\left(\mathrm{Ka}=10^{8}-10^{9}\right.$ litres $\left./ \mathrm{mole}\right)$ exprimé exclusivement sur les monocytes. Il fixe les IgG2a de souris et pourrait être l'équivalent du RFcI de souris. Son poids moléculaire (PM) de $72 \mathrm{kDa}$ est différent de celui du RFcII, de PM $40 \mathrm{kDa}$. Ce dernier a une distribution cellulaire plus large et il est exprimé sur les monocytes, neutrophiles, éosinophiles, plaquettes et lymphocytes. Il fixe les IgGl et les IgG2b de souris. Le troisième type de $R F c \gamma$ appelé $R F c_{10}$ est de très faible affinité pour l'IgG et a un poids moléculaire de 50-70 kDa. Ces trois types de récepteurs fixent les IgGl et IgG3 humaines [8]. Chez la souris, un $\mathrm{RF} c \gamma$ a été récemment purifié par chromatographie d'affinité, l'ADNc isolé et séquencé à partir d'ARNm de la ligné WEHI-3B. Ce dernier code pour un polypeptide de $37 \mathrm{kDa}$ présentant les caractéristiques des protéines membranaires [9]. Les PM des RFc $\gamma$ murins glycosylés sont situés entre 47 et $70 \mathrm{kDa}$.

En bref, les cellules du système immunitaire sont équipées de structures capables d'interagir avec la portion Fc des anticorps. Si nous revenons au phénomène de régulation isotypique décrit plus haut (figure 1), une question se pose : si toutes les cellules possèdent ces différentes structures, d'où vient la spécificité de la régulation isotypique ? D'où vient le signal capable de déclencher une cascade d'événements dont l'effet final est une perturbation du taux de synthèse d'un seul isotype ? Ce sont les travaux de J. Yodoi, K. Ishizaka et al., d'une part, et de notre laboratoire d'autre part, qui ont apporté des réponses à ces questions. Ce 
groupe de chercheurs a montré que l'interaction de lymphocytes $\mathrm{T}$ activés avec des $\operatorname{IgE}$ provoque une augmentation du taux d'expression des RFc [10]. Ce phénomène d' "induction " d'expression de RFce a été observé depuis pour tous les isotypes. Ainsi avons-nous montré que l'incubation de clones de lymphocytes $T$ porteurs à la fois de RFc $\gamma$, RFc $\alpha$, RFce et RFc $\mu$ avec chaque isotype induit une augmentation d'expression du récepteur spécifique de cet isotype [11]. Le signal déclenchant les événements régulateurs est donc apporté par la portion Fc de l'anticorps. Des travaux récents ont montré qu'il n'est efficace que sous forme dimérique et que s'il est reçu par une cellule préalablement activée (par un antigène ou par une lymphokine).

Ce phénomène d'induction fut également observé in vivo à la fois chez la souris et chez l'homme, dans des situations de perturbation des taux d'Ig sériques. Ainsi Lynch et al. montrèrent chez des souris porteuses de myélome l'existence d'un grand nombre de lymphocytes $\mathrm{T}$ qui exprimaient des récepteurs spécifiques de l'isotype [3]. Le même phénomène se produit chez les malades ayant un myélome. Par ailleurs le taux de lymphocytes $T$ porteurs de RFc $\alpha$ est augmenté dans la maladie de Berger, où le taux d'IgA sériques est trop élevé par rapport à la moyenne, celui de lymphocytes $\mathrm{T}$ à RFce est également augmenté dans le cas d'hyperproduction d'IgE. Si ces expériences démontrent la spécificité de l'induction de l'expression des RFc, elles apportent également une autre information. Alors que la quasi-totalité des cellules du système immunitaire exprime des RFc, il semble que, chez la souris, le type cellulaire dont le taux d'expression de RFc est perturbé par l'interaction avec l'isotype est essentiellement le lymphocyte $T$. Le mécanisme de cette modification d'expression n'est pas encore éclairci.

Des substances, autres que les Ig peuvent modifier l'expression des $m / s n^{\circ} 3$ vol. 3 , mars 87
RFc. Ainsi est-il connu depuis longtemps que le traitement des lymphocytes et des macrophages par les interférons induit une augmentation d'expression des RFc $\gamma$ [12]. Sur les lymphocytes $\mathrm{T}$, l'addition d'interféron $\gamma$ provoque également une diminution de l'expression de RFc $\alpha$ [6]. Il a été montré récemment que le taux de RFce des lymphocytes B est augmenté par l'addition du facteur de croissance appelé BSF1 (B cell stimulating Factor), alors que l'expression des RFc $\delta$ sur les lymphocytes $T$ est augmentée par l'interleukine 2.

L'expression des RFc sur la membrane des cellules du système immunitaire n'est donc pas permanente. Elle est la résultante des interactions que ces cellules ont avec les Ig et les médiateurs environnants.

\section{Les immunoglobulin- binding factors (IBF)}

Les IBF constituent une famille de lymphokines se liant spécifiquement au fragment Fc des Ig. Des IBF spécifiques des IgG (IgG-BF), $\operatorname{IgE}(\operatorname{IgE}-\mathrm{BF}), \operatorname{Ig} \mathrm{A}(\operatorname{IgA}-\mathrm{BF})$ ont été décrits chez la souris, le rat et l'homme [13]. Les IBF régulent respectivement la production d'IgG, d'IgE et d'IgA soit en la diminuant (IgG-BF, IgE-BF et IgA-BF suppresseurs), soit parfois en l'augmentant (IgE-BF et IgA$\mathrm{BF}$ amplificateurs). Les IgG-BF spécifiques de certaines sousclasses d'IgG sont capables de perturber la sécrétion de ces mêmes sous-classes. Ainsi l'IgG1-BF se lie aux IgG1 et bloque la production des IgG1 alors que l'IgG2a-BF agit sur les IgG2a et IgG2b sans modifier le taux d'IgG1 [14]. Dans des expériences in vitro, l'activité biologique de ces lymphokines s'exprime à de très faibles concentrations. Ainsi, 1 pg d'IgG-BF (soit 1,5 × 10 molécules) peut inhiber la production d'anticorps de 4000 plasmocytes (un plasmocyte sécrète 1 à $2 \mathrm{pg} \mathrm{d}^{\prime} \mathrm{Ac}$, soit $4 \times 10^{6}$ molécules à l'heure).

Étant donné la spécificité des IBF pour le fragment Fc des Ig, les relations entre ce type de lymphokines et les récepteurs de Fc de même spécificité ont été analysées. Nous avons observé que l'incubation de lymphocytes $\mathrm{T}$ porteurs de $R F c \gamma$, pendant trois heures, dans une solution saline physiologique, provoque une diminution d'expression de RFc membranaire, alors que de l'IgG-BF apparaît dans le surnageant des cellules [15]. L'incubation, dans des conditions similaires, de lymphocytes $\mathrm{T}$ ne portant pas de RFc $\gamma$, ne provoque pas l'apparition d'IgG-BF. L'ensemble de ces arguments nous a suggéré que l'IgG-BF pouvait être la forme soluble du RFc $\gamma$. Ce point se trouve conforté par divers résultats expérimentaux obtenus dans d'autres systèmes. Chez l'homme, la production d'IgG-BF peut être obtenue dans les mêmes conditions et s'accompagne de relargage de $R F c \gamma$.

Les IBF sont des glycoprotéines réagissant avec des AcM antiRFc. Après chromatographie, leur activité biologique apparaît associée à différentes molécules variables par leur poids moléculaire et leur charge [6]. L'hétérogénéité de taille (des poids moléculaires de 75-80 kDa, 35-40 $\mathrm{kDa}$ et $15-20$ $\mathrm{kDa}$ ont été identifiés en présence d'agents réducteurs dans le cas de l'IgG-BF et de l'IgE-BF) reflète probablement l'existence de produits de dégradation dont certains pourraient provenir du RFc. A l'heure actuelle, les IBF n'ont pas encore été purifiés à l'homogénéité, ni séquencés. En ce qui concerne l'IgE-BF, la portion glucidique de la molécule semble jouer un rôle essentiel dans l'activité biologique du facteur. Les différences biochimiques entre l'IgE-BF amplificateur qui augmente la production d' $I g E$ et l'IgE-BF suppresseur qui la diminue résident dans une différence de glycosylation. Le facteur suppresseur est $O$ glycosylé et le facteur amplificateur à la fois $\mathbf{N}$ et O glycosylé, alors que leurs chaînes polypeptidiques sont les mêmes [16]. La situation est différente dans le cas de l'IgG-BF, les molécules non glycosylées étant 
suppressives. Les expériences de clonage génétique de l'IgE-BF murin ont abouti à l'isolement de gènes de rétrovirus présentant des activités biologiques similaires à celle du facteur [17]. Ce point reste, pour l'instant, inexpliqué. Comme nous l'avons indiqué plus haut, les IBF pourraient représenter une forme soluble ou sécrétoire des RFc. Puisque les cellules du système immunitaire expriment des RFc pour tous les isotypes, produisent-elles en permanence des IBF pour tous les isotypes? Tel ne semble pas être le cas. Nous avons montré que l'interaction d'un lymphocyte $\mathrm{T}$ porteur de RFc $\gamma$, RFc $\alpha$, RFc $\epsilon$ et $\mathrm{RF} c \mu$ avec des IgA par exemple, induit une production importante d'IgA-BF alors que celle d'IgG$B F$ est quasi nulle. Encore une fois, ce sont donc les Ig qui donnent une spécificité isotypique au système de régulation. Leur interaction avec les cellules exprimant des RFc induit non seulement une augmentation de l'expression du RFc correspondant, mais aussi celle de la production de l'IBF de spécificité correspondante.
La grande majorité des cellules du système immunitaire exprime des RFc. Peuvent-elles toutes produire des IBF régulateurs? Chez l'homme, les travaux de J.-P. Revillard et al. ont montré que des neutrophiles, des monocytes, des lymphocytes B peuvent produire des IBF [18]. Chez les rongeurs, pour des raisons inexpliquées jusqu'à maintenant, la production d'IBF semble être restreinte aux lymphocytes $T$.

Les cellules cibles des IBF suppresseurs chez la souris sont les lymphocytes B. Ainsi l'IgG-BF, l'IgE-BF et l'IgA-BF sont capables d'inhiber la production d'Ig par des hybridomes B in vitro et, dans certains cas, leur prolifération est également affectée [19] (figure 3). L'IgG-BF peut inhiber des réponses anticorps vis-à-vis d'antigènes indépendants des cellules T, comme le LPS, aussi bien que des réponses anticorps thymodépendantes.

Les lymphokines agissent par l'intermédiaire de récepteurs exprimés sur les cellules cibles, tels le récepteur pour l'interféron dans le cas de l'interféron, ou le récepteur pour l'interleukine 2 dans le cas de l'IL2. En ce qui concerne les IBF, il semble que la structure membranaire cible du facteur soit l'immunoglobuline membranaire du même isotype que l'Ig sécrétée par les cellules B. Ainsi, par exemple, les lymphocytes $B$ ayant commuté pour synthétiser des anticorps de classe IgG expriment sur leur membrane des Ig de même spécificité qui peuvent servir de cible à l'IgG-BF. Il en est de même pour les IgA-BF ou les $\operatorname{IgE}$-BF qui agissent respectivement sur les cellules $B$ ayant commuté pour synthétiser des IgA ou des IgE.

Chez l'homme, le mécanisme d'action des IBF pourrait être plus complexe. Ainsi, J.-P. Revillard et al. ont montré que des cellules $\mathrm{T}$ sont parfois nécessaires à l'action de l'IBF [20].

\section{Les circuits isotypiques et leurs dérèglements}

L'ensemble des données décrites dans les deux paragraphes précédents nous a amenés à décrire un
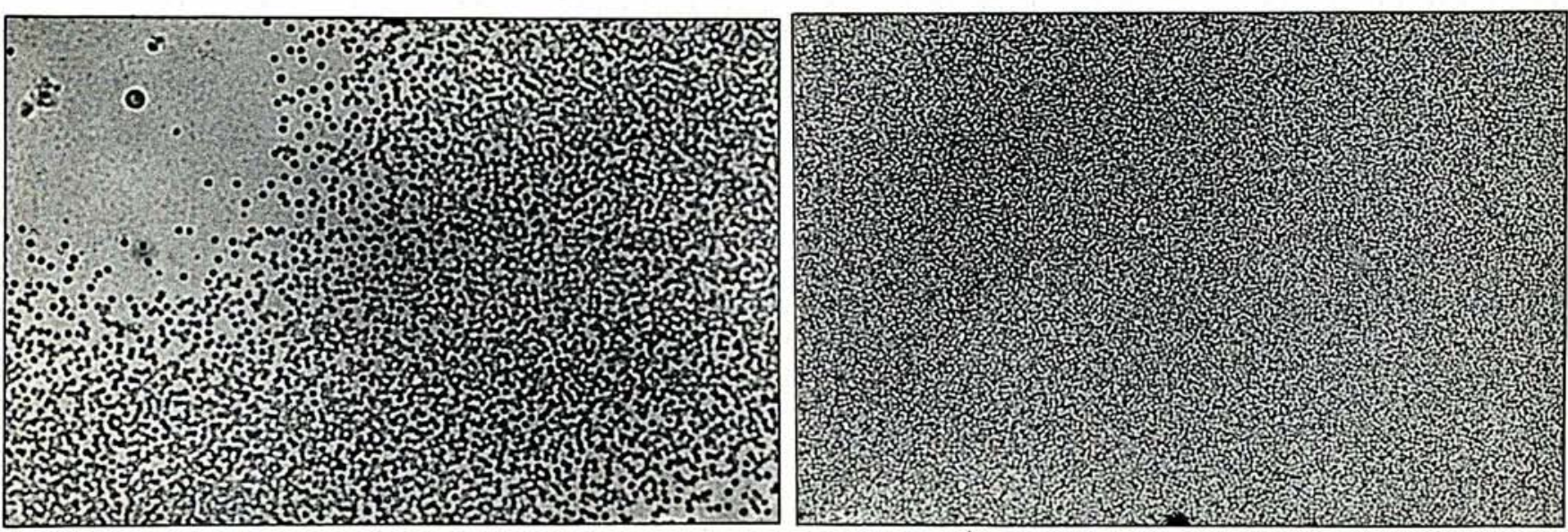

A

Figure 3. L'IBF inhiba la production d'anticorps par des cellules d'hybridome B. Mise en évidence par la technique d'hémolyse. A. Production d'anticorps en l'absence d'IBF : les cellules d'hybridome $B$ produisent des anticorps anti-GRM. En présence de complément, une zone de lyse du tapis de GRM est visible autour d'une cellule B. B. Inhibition de la production d'anticorps par I'IBF : les cellules $B$ traitées pendant $18 \mathrm{~h}$ par de l'IBF ne synthétisent plus $d^{\prime}$ anticorps; on ne voit plus de plage de lyse autour de la cellule B [19]. 


\begin{tabular}{|c|c|c|c|}
\hline \multicolumn{4}{|c|}{$\begin{array}{c}\text { Tableau ॥ } \\
\text { SITUATIONS PATHOLOGIQUES DE PERTURBATION } \\
\text { DU SYSTĖME DE RÉGULATION ISOTYPIQUE }\end{array}$} \\
\hline Pathologie & Sérum lg & RFc & IBF \\
\hline $\begin{aligned} & \text { Myélomes }^{0 *} \text { à } \lg \mathrm{A} \\
& \text { à } \operatorname{lgG} \\
& \text { à } \lg \mathrm{E} \\
& \text { a } \operatorname{lgM} \\
& \text { à } \lg D\end{aligned}$ & $\begin{array}{l}\lg A \\
\lg G \mid \\
\lg E \\
\lg M \\
\lg D\end{array}$ & $\begin{array}{l}\alpha \\
T \gamma \\
T \epsilon \\
T \mu \\
T \delta\end{array}$ & $\begin{array}{l}\text { IgA-BF suppresseur } \mid \\
\text { Effet suppresseur des IgG-BF| } \\
\text { N.D. } \\
\text { N.D. } \\
\text { IgD-BF amplificateur } \mid\end{array}$ \\
\hline Syndrome hyper $\lg \mathrm{E}^{\circ}$ & $\operatorname{IgE} \mid$ & $T \in \mid$ & |gE-BF amplificateur | \\
\hline Allergies $^{0}$ & $\lg \mathrm{E} \mid$ & $T \epsilon \mid$ & N.D. \\
\hline Maladie de Berger ${ }^{\circ}$ & $\lg A \mid$ & $T \alpha \mid$ & N.D. \\
\hline Cirrhoses $^{\circ}$ & $\lg G \mid$ & $T_{\gamma l}$ & IgG-BF I \\
\hline $\begin{array}{l}\text { Parasitoses } \\
- \text { Nippostrongylus*,** } \\
\text { brasiliensis }\end{array}$ & $|g E|$ & $T \epsilon 1$ & $\lg E-B F \mid$ \\
\hline $\begin{array}{l}\text { Néphrite par intoxica- } \\
\text { tion au mercure }{ }^{*}\end{array}$ & $\lg \mathrm{E} \mid$ & $T \epsilon \uparrow$ & $\operatorname{IgE}-\mathrm{BF} \mid$ \\
\hline
\end{tabular}

Chez I'homme $\left({ }^{\circ}\right)$, la souris $\left({ }^{*}\right)$ et le rat $\left({ }^{*}\right)$ [8].

| Taux augmenté par rapport à la normale. N.D. = non déterminé.

modèle expliquant la régulation de la production des isotypes [21]. Ce modèle est schématisé dans la figure 1 et est applicable à tous les isotypes. Les lymphocytes B produisent des anticorps d'un isotype donné, par exemple IgG en réponse secondaire. Ces anticorps vont interagir avec les $\mathrm{RFc} \gamma$ des lymphocytes $T$, ce qui engendre une augmentation de leur expression et le relargage de quantités importantes d'IgG-BF. Celui-ci va agir sur les lymphocytes B et inhiber leur synthèse d'IgG. Des substances telles que les interférons peuvent également agir sur le système, mais de façon non spécifique d'isotype. Ainsi, l'interféron peut réguler la production d'anticorps, soit en l'augmentant, soit en la diminuant. D'autres facteurs intervenant dans la glycosylation des protéines par l'intermédiaire de la phospholipase A2 (qui stimule les $\mathrm{N}$-glycosyl-transférases) paraissent également importants dans le cas de la régulation de la synthèse des $\operatorname{IgE}$, la lipocortine* déplaçant le système de régulation vers la formation d'IgE-BF suppresseur et la bradykinine vers celui d'IgE-BF amplificateur.

$m / s n^{\circ} 3$ ool. $3, \operatorname{mars} 87$

\section{Summary}

Isotype production by $\mathrm{B}$ cells is the consequence of a series of interactions including immunoglobulins (Ig), T cells, receptors for the Fc portion of immunoglobulins, FcR, and immunoglobulin-binding factors (IBF). The expression of FcR for a given isotype on $T$ cells is dependent on environmental factors such as Ig or lymphokines. IBF are FcR related glycoproteins produced by FcR-positive $T$ cells. They bind to the Fc portion of Ig and up or down regulate $\mathrm{Ig}$ production in an isotype specific manner. IBF specific for $\operatorname{IgG}, \operatorname{IgA}$ and $\operatorname{IgE}$ regulate the production of $\operatorname{IgG}, \operatorname{IgA}$ or $\operatorname{IgE}$ respectively. Treatment of $\mathrm{T}$ cells with Ig induces IBF production.

A model for the regulation of isotype production is described. It involves : the interaction of $\mathrm{Ig}$ of a given isotype with $\mathrm{FcR}$ positive $T$ cells, a subsequent increase of the expression of FcR and the production of IBF specific for this isotype by $T$ cells and, finally, an up or down regulation of the secretion of this isotype by B cells. modulation du taux de production d'une classe d'Ig dans des normes physiologiques. Des dérèglements de cette régulation peuvent se produire. Certains sont indiqués dans le Tableau II. Ce sont des situations où une augmentation importante du taux d'un isotype circulant, provoquée par exemple par le développement d'un myélome, d'une parasitose ou de réactions allergiques, s'est trouvée toujours accompagnée d'une augmentation du taux de lymphocytes $\mathrm{T}$ porteurs de RFc correspondants. Dans tous les cas où cela a été recherché, une perturbation de la production des IBF a été observée. Il est possible par ailleurs que certains dysfonctionnements de la production d'un isotype soit la résultante d'une dérégulation de la production d'un IBF. Le syndrome d'hyper IgE pourrait en être un exemple. D'autres modèles physiopathologiques sont actuellement à l'étude
- La lipocortine est une protéine induite par les corticoides, inhibant la phospholipase $A_{2}$ et, par conséquent, la $N$-glycosylation des protéines.

\section{TIRÉS A PART}

C. Sautès : Laboratoire d'immunologie cellulaire et clinique, Unité Inserm U.255, Institut Curie, 26, rue d'Ulm, 75005 Paris. 